IP Periodica Polytechnica

Chemical Engineering

58(Sup), pp. 53-59, 2014

DOI: $\underline{10.3311 / P P c h .7188}$

http://www.pp.bme.hu/ch/article/view/7188

Creative Commons Attribution (i)

RESEARCH ARTICLE

\section{A comparison of contact angle measure- ment results obtained on bare, treated, and coated alloy samples by both dy- namic sessile drop and Wilhelmy method}

Loránd Románszki / Miklós Mohos / Judit Telegdi / Zsófia Keresztes / Lajos Nyikos

RECEIVED 1 November 2012; ACCepted 11 March 2013

\section{Abstract}

The goal of this work was to compare the performance of our home-built dynamic sessile drop contact angle (CA) goniometer with our NIMA Dynamic Surface Tensiometer. Water CA measurements on different alloy samples (aluminium brass, copper-nickel 70/30, stainless steel 304) have been carried out by 1) sessile drop and 2) Wilhelmy plate method. Different sets of substrates were i) cleaned; ii) cleaned and pre-treated; iii) cleaned, pre-treated and coated with atactic polystyrene. During these experiments, the main features of the two setups in connection with our sample properties were understood. We therefore found it desirable and justified to sum up our findings.

\section{Keywords}

contact angle $\cdot$ sessile drop $\cdot$ Wilhelmy $\cdot$ copper-nickel $\cdot$ aluminium brass $\cdot$ stainless steel

\section{Loránd Románszk}

Department of Interfaces and Surface Modification, Institute of Materials and Environmental Chemistry, Research Centre for Natural Sciences, Hungarian Academy of Sciences Pusztaszeri út 59-67., H-1025 Budapest, Hungary

e-mail: romanszki.lorand@ttk.mta.hu

\section{Miklós Mohos}

Judit Telegdi

Zsófia Keresztes

Lajos Nyikos

Department of Interfaces and Surface Modification, Institute of Materials and Environmental Chemistry, Research Centre for Natural Sciences, Hungarian Academy of Sciences Pusztaszeri út 59-67., H-1025 Budapest, Hungary

\section{Introduction}

Contact angle (CA) measurement is perhaps the simplest routine measurement to give information about the success of a coating procedure. The principles have been formulated long time ago by Young [1]. There are many different techniques of measuring CAs, but the most common two are the sessile drop and the Wilhelmy plate technique (Figure 1).

The first method is direct, as the liquid droplet is placed on the surface to be characterized and the image is captured and the angle of contact formed at the gas/liquid/solid interfaces is evaluated. The method can be used in both static and dynamic mode. During this latter, the drop is continuously increased by adding liquid to it and the CA is measured in function of the drop volume. The highest possible angle measured is regarded as the advancing CA. Then, the drop size is gradually decreased by withdrawing liquid from it, while the CA is again recorded in function of the volume. The lowest possible measured angle is taken as the receding CA.

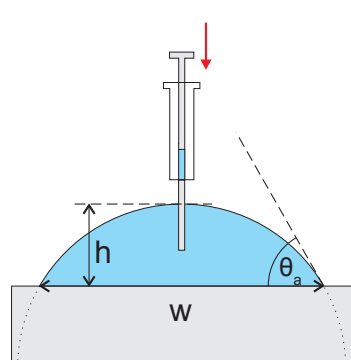

$\theta_{\mathrm{a}}=2 \cdot \arctan (2 \mathrm{~h} / \mathrm{w})$

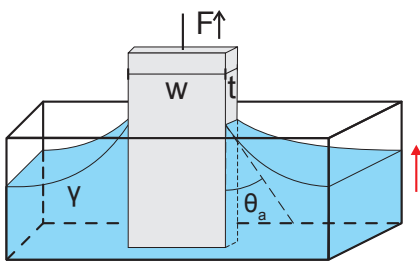

$\theta=\arccos [F / 2(w+t) y]$
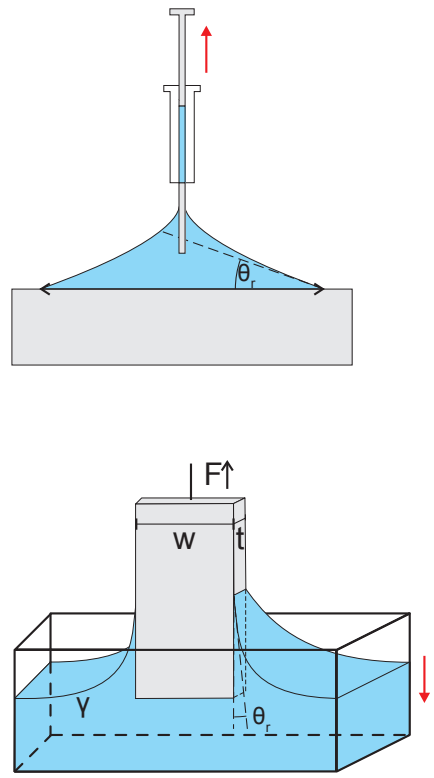

Fig. 1. Schematic illustration of the sessile drop (top) and Wilhelmy (bottom) CA measurement principle 
The necessity of this separate measurement of advancing and receding CA cannot be emphasized enough. Unfortunately, in the literature often can be read statements like 'the CA of the surface was $78.5^{\circ}$, without being specified which CA was measured. Measuring only static CAs is generally misleading, as the surface properties are sometimes reflected mainly in the receding angles. Therefore, a scientifically more correct characterization of a surface is to provide both advancing and receding CAs, and even the difference of these two, i.e. the CA hysteresis.

The Young equilibrium CA, which would, in an idealised case, unequivocally characterise a surface, is practically not accessible by any means. Instead, it has to be calculated, and often just approximated, from the measurement data. Furthermore, the static CAs measured are definitely not identical to this theoretical, ideal CA [2]. There are different, accepted methods of retrieving equilibrium CAs. The simplest approximation is to take the arithmetical average of the advancing and receding angle. A somewhat better approximation is to take the average of the cosines. Tadmor's theoretically even more precise, yet considerably cumbersome expression is also widely used nowadays (Equations 1-3) [3].

$$
\begin{gathered}
\theta_{\text {eq }}=\arccos \left(\frac{\Gamma_{\mathrm{a}} \cos \theta_{\mathrm{a}}+\Gamma_{\mathrm{r}} \cos \theta_{\mathrm{r}}}{\Gamma_{\mathrm{a}}+\Gamma_{\mathrm{r}}}\right) \\
\Gamma_{\mathrm{a}} \equiv \sqrt[3]{\frac{\sin ^{3} \theta_{\mathrm{a}}}{2-3 \cos \theta_{\mathrm{a}}+\cos ^{3} \theta_{\mathrm{a}}}} \\
\Gamma_{\mathrm{r}} \equiv \sqrt[3]{\frac{\sin ^{3} \theta_{\mathrm{r}}}{2-3 \cos \theta_{\mathrm{r}}+\cos ^{3} \theta_{\mathrm{r}}}}
\end{gathered}
$$

The principles of Wilhelmy plate technique are also well established [4]. The substrate to be studied is perpendicularly immersed to and withdrawn from the measurement liquid with a known rate. At each position, the apparent weight change of the substrate due to the interplay between the Archimedes' buoyancy force and the surface tension of the liquid acting to the substrate is recorded. Knowing the surface tension and the perimeter of the substrate, the CA at each position can be calculated. The technique, thus, is dynamic by nature, and the advancing and receding angles are determined similarly as described above. One big advantage of the method is that several immersion cycles can be recorded over relatively large distances (some centimetres), thus the in situ changes due to layer break or reorganization can be studied in time and space. However, its big drawback is that only samples with regular profiles can be accurately measured: typically fibres, rods and thin plates (as also the name suggests). In this latter case, the two sides must be homogenous, both chemically and regarding roughness.

As far as roughness is concerned, this affects the measured CA. The general rule is that hydrophilic surfaces (of CA less than $90^{\circ}$ ) become more hydrophilic, while hydrophobic surfaces (of CA more than $90^{\circ}$ ) become more hydrophobic upon roughening. This is another way of formulating Wenzel's law [5], which considers the increased surface area of a rough sample as compared to a chemically identical smooth one. Later it has been pointed out that not the interfacial area, but the contact line is what determines the CA [6]. Yet, Wenzel's equation has become classical and it is still widely used.

The surfaces are often composed of high and low energy domains. An example would be a metal oxide with a loosely packed self-assembled monolayer (SAM) on it. In this case, the metal oxide is mainly reflected in the receding $\mathrm{CA}$, while the SAM contributes to the advancing CA. The Cassie-Baxter theory [7] describes the overall CA of such composite surfaces as the sum of CAs of the individual component types, each weighted with its relative surface coverage.

In the simplest case, one measuring liquid is used, usually water. From the measurement data, it is possible to extract surface free energy values using Neumann's - nowadays disputed - equations of state of limited validity [8]. Pászli's Individual Representation Approach is an even simpler model, which apparently works satisfactory on hydrophobic surfaces [9]. This model operates with individual substantial parameters of $(\mathrm{mN} / \mathrm{m})^{-2} \equiv\left(\mathrm{mJ} / \mathrm{m}^{2}\right)^{-2}$ dimensions, and all interfacial tensions, including the solid/gas interfacial tension, i.e. the surface free energy, are defined as products of the corresponding two parameters, wherefrom the missing quantities can be deducted.

For a more profound measurement, Zisman's method can be followed [10]. A set of liquids with different surface tensions are used and the cosine of the measured angles plotted as a function of the liquids' surface tensions. Then, the surface can be characterised by its so-called critical surface tension. A liquid which surface tension matches this value is the highest surface tension liquid which still can completely wet that respective surface (produces a zero CA). Liquids with lower surface tension than this will completely wet that surface, while liquids with higher surface tensions will only partially wet it (producing a $\mathrm{CA}>0^{\circ}$ ).

Perhaps the most recognized and extensive method is the one suggested by van Oss et al. [11]. According to them, the surface free energy has additive and non-additive components and must be separately determined by measuring CAs of at least three different liquids on the respective substrate, of which at least two should be polar and capable of forming hydrogen bonds, and at least one should be non-polar. The merit of the theory and the method is that can explain different behaviour of surfaces having otherwise the same overall surface free energy. 
The target materials of our research are aluminium brass (Al-brass), copper-nickel 70/30 (CuNi) and stainless steel 304 (SS). These are important alloys used for the fabrication of heat exchanger tubes $[12,13]$. These tubes suffer from biofouling and the consecutive microbially-induced corrosion [14]. Nanolayers are potential solutions for the mitigation of the problem [15-22], and the success of the nanolayer coating procedure is routinely checked by CA measurements [23].

In this study, our aim was to compare the performance of our new homebuilt dynamic sessile drop CA goniometer with that of our dynamic surface tensiometer (Nima).

\section{Materials and methods}

Substrates. The original samples were sections of condenser tubes, and the curvature presented a problem in the objective evaluation of the measured CAs. (A mathematical correction derived for the curvature will be presented later elsewhere.) Therefore the samples were flattened in a press. The samples were not polished and had a surface roughness of 200-300 nm before flattening, depending on alloy type and side, generally the inner side being smoother.

Cleaning, pre-treatment and coating. Samples were successively

1) acetone-cleaned;

2) pre-treated for $5 \mathrm{~min}$ in $14 \mathrm{mM} \mathrm{HNO}_{3}$;

3) followed by $5 \mathrm{~min}$ in $5 \% \mathrm{H}_{2} \mathrm{O}_{2}$ ultrasonic bath;

4) and finally dip-coated by immersing/withdrawing into/from $10 \mathrm{~g} / 1$ polystyrene (atactic; $M_{\mathrm{n}}=88,700 ; Ð=2.84$ ) solutions in dichloromethane with a rate of $5 \mathrm{~mm} / \mathrm{s}$.

The concordant results of independent AFM and spectroscopic ellipsometric measurements showed that the thickness of the coatings was approx. $90 \mathrm{~nm}$.

Sessile drop measurements were carried out using a home-built measurement device equipped with a Hamilton micropipette and a CCD camera. The volume of water droplets was increased and decreased by the aid of a programmable syringe pump (New Era Pump Systems Inc.) by a constant rate of $20 \mu \mathrm{l} / \mathrm{min}$, in $1 \mu \mathrm{l}$ steps. Advancing and receding CAs were determined by geometrical evaluation of the drops. Working with sufficiently small droplets (a few $\mu \mathrm{l}$ ), a spherical cap drop shape was assumed [24]. The CAs were determined by taking the ratio of the drop height $h$ and drop base width $w$ (Figure 1).

Wilhelmy CA measurements. A digital surface tensiometer (Nima) was used. Samples were automatically dipped into and pulled out from water with a rate of $10 \mathrm{~mm} / \mathrm{min}$, while the apparent weight change of the target sample was registered. The CAs were retrieved by inputting the cross sectional dimensions of the samples and the surface tension of the water. Ultrapure water (MilliQ) was used as a measurement liquid.

\section{Results and discussion}

Typical hysteresis curves obtained in both types of measurements are presented in Figure 2.

In the case of sessile drop measurements, the advancing CA (A) was designated as the one corresponding to the plateau region. A similar plateau in the receding branch could not be observed. Therefore the lowest measured CA was designated as receding CA (R). The small arrows in the Figure show the path of the droplet's history.

In the Wilhelmy measurements, both plateaus appeared, thus the receding CAs were designated as the lower plateau value. The small arrows on the Figure indicate where the sample first came into contact with water surface during immersion, respectively the point where it finally detached from the water surface during withdrawal.

Three parallel samples of each type were measured, and the average values and standard errors were calculated. In the case of the sessile drop method, measurements were done on both sides of the samples. CA hystereses $(\mathrm{H})$ were calculated as the difference between A and R. Equilibrium CAs (E) were calculated using Tadmor's equation (Equations 1-3). Averaging was done in both cases similarly.
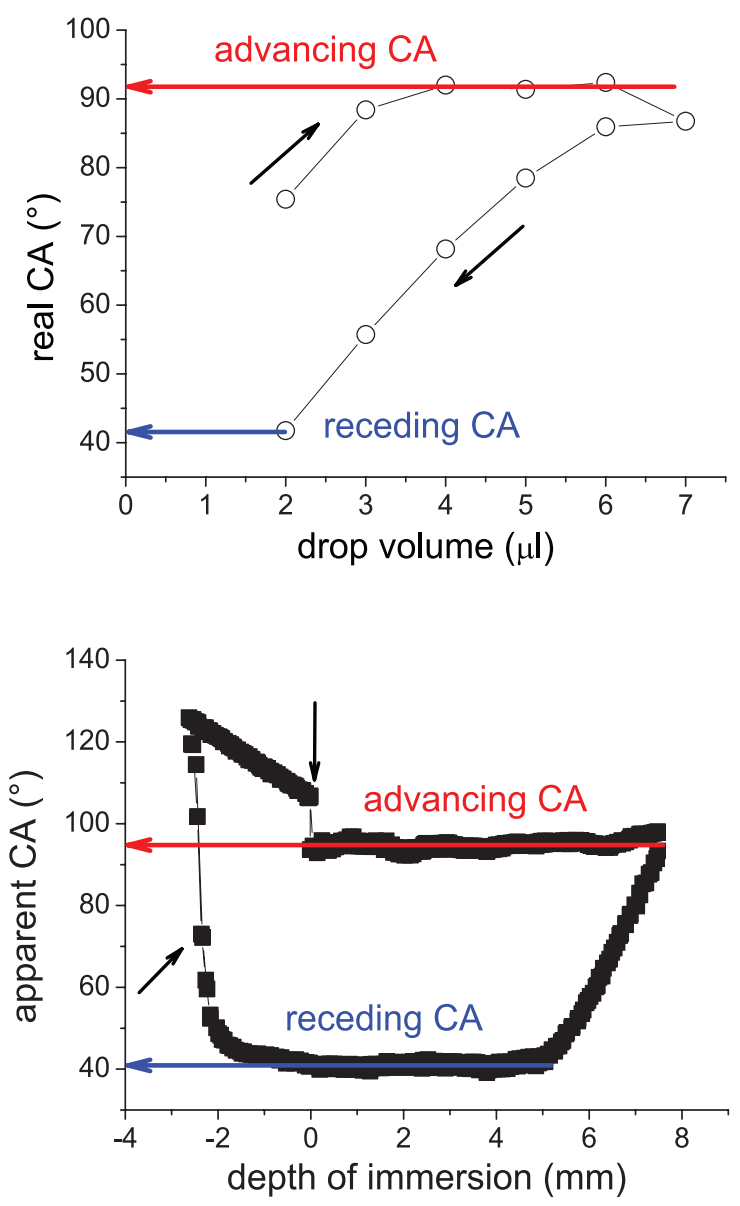

Fig. 2. Typical hysteresis curves obtained in the sessile drop (top) and the Wilhelmy (bottom) measurements and the designation of the advancing and receding $\mathrm{CAs}$ 
All CA values are plotted in Figure 3. In each series, the first point corresponds to the acetone-cleaned sample, the second one to the $\mathrm{HNO}_{3}$-treated sample, the third one to the $\mathrm{H}_{2} \mathrm{O}_{2}$-treated sample and the fourth one to the PS-coated sample. The following observations can be made:

In the case of sessile drop results, average advancing CAs generally decrease after $\mathrm{HNO}_{3}$-treatment, decrease further after $\mathrm{H}_{2} \mathrm{O}_{2}$-treatment and markedly increase after PS-coating, the outer surfaces generally showing somewhat higher advancing CAs than the inner ones. As suggested by our unpublished XPS results and by others too [25], upon $\mathrm{HNO}_{3}$-treatment certain surface oxides are etched away, while upon $\mathrm{H}_{2} \mathrm{O}_{2}$-treatment they are reconstructed, without the bulk of the material being affected. The changes in the wetting behaviour can be correlated with the disappearance and reappearance of these surface oxides of different types. The much larger advancing CAs of the PS coated samples are in agreement with the literature values [26] and indicate that the coating process was successful. The error bars of the advancing CAs are large for the first three process steps, but decrease considerably after the last - coating step, irrespectively of the alloy type, indicating not only a more hydrophobic, but also an energetically more uniform surface.

Receding CAs are more or less the same after the first three processing steps (acetone, $\mathrm{HNO}_{3}, \mathrm{H}_{2} \mathrm{O}_{2}$ ), but increase considerably after the PS-coating, for all alloy types. The receding angles after PS-coating are somewhat higher on the SS samples than on other alloys, suggesting that PS chain density is higher on treated SS surface than on other alloys.
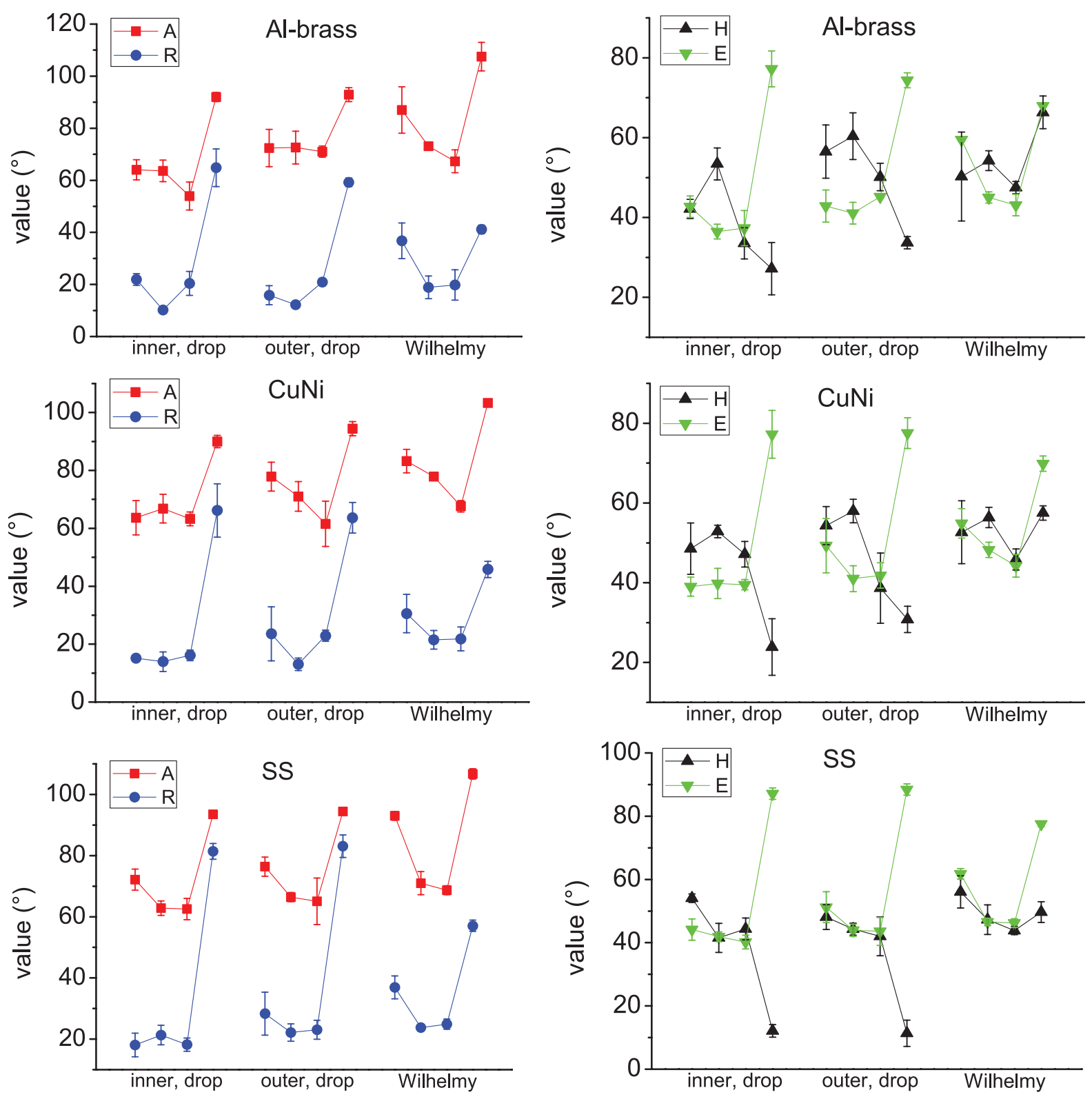

Fig. 3. Series of CA measurement results obtained on Al-brass, CuNi and SS samples, inner and outer tube side by sessile drop, as well as Wilhelmy method. Explanation in the text. 
Consequently, understandably, the CA hystereses are generally high in the case of cleaned and $\mathrm{HNO}_{3}$-treated samples, drop after the $\mathrm{H}_{2} \mathrm{O}_{2}$ treatment and decrease much more after the PS coating, for all alloy types. The hystereses measured on the outer surfaces of Al-brass and CuNi samples in all processing stages were slightly higher than the ones measured on the inner surfaces. In the case of SS samples, no such significant difference was observed. The hysteresis is generally slightly decreasing in the Al-brass $>\mathrm{CuNi}>\mathrm{SS}$ order for all sides and processing stages, becoming conspicuous mainly in the PS-coated series.
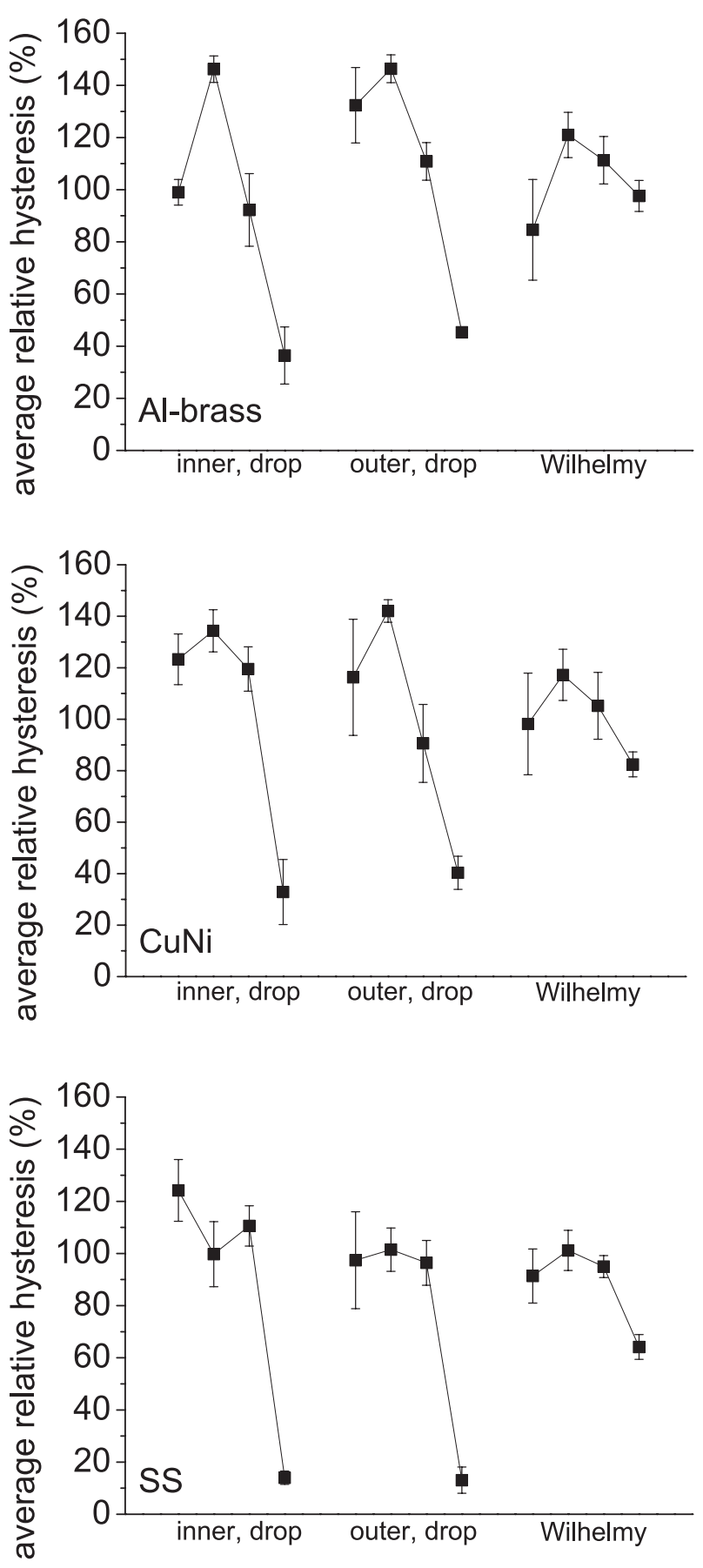

Fig. 4. Relative CA hystereses measured on the three alloy substrates in each of the four processing stages by sessile drop, respectively Wilhelmy method
Accordingly, the trend in the equilibrium CAs follows that of the advancing and receding ones, showing a pronounced increase in the PS-coated series. These values are almost identical on the inner and outer surfaces, probably indicating the same, good quality, homogeneous PS layer on both sides. For these PS-coated samples, Al-brass and CuNi ones showed the same equilibrium CA values, while the CAs calculated on SS samples were slightly, but significantly higher. Also the errors were lower. These findings suggest that PS might adhere either 1) better; or 2) in a more compact form on the pre-treated SS surface (practically $\mathrm{Cr}_{2} \mathrm{O}_{3}$ ) than on either Al-brass or $\mathrm{CuNi}$, as argued above. All these together indicate that for the formation of a good quality, dense PS layer, the chemical composition of the substrate surface is the deciding factor and not its physical heterogeneity (roughness).

In the Wilhelmy-type CA measurements the general observations are the followings:

The trend in the advancing angles for the first three processing stages (cleaning, $\mathrm{HNO}_{3}, \mathrm{H}_{2} \mathrm{O}_{2}$ ) is similar to the one found in the sessile drop measurements. However, CAs are somewhat larger (in the most extreme cases, by about $15^{\circ}$ ) and are closer to the sessile drop results measured on the outer sides. As the wetting is more influenced by the hydrophobic domains of a surface, this fact can be explained by the dominance of the somewhat more hydrophobic outer sides during the immersion.

Based on this argument, one would expect the measured receding angles to be accordingly closer to the sessile drop experimental results of the slightly more hydrophilic inner sides. This is, however, not the case, since the magnitudes of the receding angles are closer to the more hydrophobic outer surfaces. To resolve this inconsistency, we must assume that the roughness difference of the two sides plays non-negligible role in both wetting and dewetting.

On the other hand, the PS-coated surfaces showed much lower receding CAs than the corresponding sessile drop measurement values. The reason can be understood from Figure 2. In the Wilhelmy experiments, a real receding CA plateau is reached, whereas in our sessile drop measurements we could only mark out the lowest measurable receding CA. This is due to the dimensions and the shape of the needle used, and there are indications that this artefact would be eliminated upon perfecting these parameters. Until then, all receding CAs and all other values calculated thereof are probably higher than the real ones.

Thus, as a consequence, CA hysteresis is not decreasing, but increasing after PS-coating. However, when we compensate for the actual magnitude of the advancing and receding CAs and take the relative, rather than the absolute CA hysteresis as defined by the ratio of the absolute hysteresis and the equilibrium CA (Equation 4), the similarity of the trend to that of the sessile drop results becomes visible, although with higher values for the PS-coated samples (Figure 4).

$$
H_{\text {rel }} \equiv 100 \times \frac{H}{E}
$$


For the same reason discussed above, equilibrium CAs calculated based on the Wilhelmy method do not appear to be the average of the equilibrium CAs of the sessile drop measurements on the two different sides, as expected, but fall somewhat lower.

\section{Conclusions}

In conclusion, it can be stated that the performance of our home built sessile drop CA goniometer is good and comparable to that of our Wilhelmy-type dynamic surface tensiometer. However, the size and shape of the needle dispensing the water droplets should be further improved in order to get access to the real, minimum receding CAs. Advantages of such a CA measuring setup over the Wilhelmy type are that:

a) two, chemically and/or physically different sides of a sample can be measured separately;

b) it can be used on curved surfaces (to be discussed elsewhere later);

c) large, heavy samples can also be measured (impossible with the limited capacity of the tensiometer balance).
However, its drawbacks and difficulties compared to the Wilhelmy-type system are that:

i) it gives less reliable absolute results when the main surface properties are reflected in the receding angles, since these are more difficult to measure accurately;

ii) its precision is less reliable when measuring very wettable or very little dewettable surfaces, as very small CAs are difficult to measure.

On the other hand, an extra advantage of the Wilhelmy method is that it can quickly measure and average CAs at numerous points (actually: lines), thus giving a CA distribution over centimetres of distance along the dipping direction (providing a quasi surface free energy mapping), whereas sessile drop technique measures only in one spot of the typical dimension of a millimetre.

\section{Acknowledgement}

József Maráczi is highly acknowledged for the construction of the sessile drop contact angle measurement setup. We also thank Györgyi Szarka for the determination of molecular weight distribution of the PS source. The research leading to these results has received funding from the European Community's Seventh Framework Programme FP7/2007-2013 under grant agreement $n^{\circ} 238579$.

\section{References}

1 Young T., An essay on the cohesion of fluids. Philosophical Transactions of the Royal Society of London, 95, 65-87 (1805). DOI: $10.1098 /$ rstl.1805.0005

2 Bormashenko E., Wetting of real solid surfaces: new glance on wellknown problems. Colloid and Polymer Science, 291, 339-342 (2013). DOI: $10.1007 / \mathrm{s} 00396-012-2778-8$

3 Tadmor R., Line energy and the relation between advancing, receding, and young contact angles. Langmuir, 20(18), 7659-7664 (2004). DOI: $10.1021 / 1 \mathrm{a} 049410 \mathrm{~h}$

4 Wilhelmy L., Ueber die Abhängigkeit der Capillaritäts-Constanten des Alkohols von Substanz und Gestalt des benetzten festen Körpers. Annalen der Physik, 195(6), 177-217 (1863). DOI: $10.1002 /$ andp. 18631950602

5 Wenzel R. N., Resistance of solid surfaces to wetting by water. Industrial and Engineering Chemistry, 28(8), 988-994 (1936). DOI: $10.1021 /$ ie $50320 \mathrm{a} 024$

6 Gao L., McCarthy T. J., How Wenzel and Cassie were wrong. Langmuir, 23(7), 3762-3765 (2007). DOI: $10.1021 / 1 \mathrm{a} 062634 \mathrm{a}$

7 Cassie A. B. D., Baxter S., Wettability of porous surfaces. Transactions of the Faraday Society, 40, 546-451 (1944). DOI: $\underline{10.1039 / T F 9444000546}$

8 Neumann A. W., Good R. J., Hope C. J., Sejpal M., An equation-of-state approach to determine surface tensions of low-energy solids from contact angles. Journal of Colloid and Interface Science, 49(2), 291-304 (1974). DOI: $10.1016 / 0021-9797(74) 90365-8$
9 Pászli I., Mohammed-Ziegler I., Hórvölgyi, Z., Obtaining surface tension from contact angle data by the individual representation approach. Colloid and Polymer Science, 285(9), 1009-1018 (2007). DOI: $10.1007 / \mathrm{s} 00396-007-1646-4$

10 Zisman W. A., Influence of constitution on adhesion. Industrial and Engineering Chemistry, 55(10), 18-38 (1963). DOI: $10.1021 / \mathrm{ie} 50646 \mathrm{a} 003$

11 van Oss C. J., Good R. J., Chaundhury M. K., Additive and nonadditive surface tension components and the interpretation of contact angles. Langmuir, 4(4), 884-891 (1988). DOI: $10.1021 / 1 \mathrm{a} 00082 \mathrm{a} 018$

12 ASTM B 111 - 98: Standard specification for copper and copperalloy seamless condenser tubes and ferrule stock (2004). DOI: $10.1520 / \mathrm{B} 0111 \mathrm{M}-98 \mathrm{E} 02$

13 ASTM A249 / A249M - 04: Standard specification for welded austenitic steel boiler, superheater, heat-exchanger, and condenser tubes (2004). DOI: 10.1520/A0249_A0249M-04

14 Cristiani P., Solutions to fouling in power station condensers. Applied Thermal Engineering, 25(16), 2630-2640 (2005). DOI: $10.1016 /$ j.applthermaleng.2004.11.029

15 Telegdi J., Szabó T., Románszki L., Pávai M., The use of nano/micro-layers, selfhealing and slow release coatings to prevent corrosion and biofouling. in 'Handbook of smart coatings for materials protection' (ed.: Makhlouf A. S. H.) Woodhead Publishing Limited, Cambridge, UK, 135-182 (2014). 
16 Telegdi J., Al-Taher F., Románszki L., Kálmán E., Nanolayers in mitigation of chemically and microbiologically influenced corrosion. in 'Proceedings of the 3rd International Conference on Technological Advances of Thin Films \& Surface Coatings: THINFILMS 2006. Singapore', paper no. TCR640, 1-5 (2006)

17 Románszki L., Telegdi J., Kálmán E., Langmuir-Blodgett and selfassembled films for metal protection. in '6th International Conference of PhD Students. University of Miskolc, Hungary', 97-102 (2007).

18 Telegdi J., Románszki L., Al-Taher F., Pfeifer É., Kálmán E., Nanolayers against microbial adhesion. in 'Proceedings of the 17th International Corrosion Congress: Corrosion Control in the Service of Society. Las Vegas, Nevada, USA', 4, 2631-2639 (2008).

19 Románszki L., Telegdi J., Kálmán E., Comparative study of Langmuir-and Langmuir-Blodgett layers of amphiphilic carboxylicand hydroxamic acids. Colloids and Surfaces A: Physicochemical and Engineering Aspects, 321(1-3), 20-28 (2008).

DOI: $10.1016 /$ j.colsurfa.2008.02.046

20 Románszki L., Telegdi J., Nyikos L., Ultrathin organic coatings on copper, copper alloys and stainless steel for controlling the microbiologically influenced corrosion. Studia Universitatis Babeş-Bolyai Chemia, 57(4), 191-200 (2012).

21 Yang Y., Poleunis C., Románszki L., Telegdi J., Dupont-Gillain C. C., Adsorption of a PEO-PPO-PEO triblock copolymer on metal oxide surfaces with a view to reduce protein adsorption and further biofouling. Biofouling, 29(9), 1123-1137 (2013).

DOI: $10.1080 / 08927014.2013 .830109$

22 Románszki L., Datsenko I., May Z., Telegdi J., Nyikos L.,

Sand W., Polystyrene films as barrier layers for corrosion protection of copper and copper alloys. Bioelectrochemistry (2013).

DOI: 10.1016/j.bioelechem.2013.10.002
23 Románszki L., Mohos M., Telegdi J., Nyikos L., Contact angle measurement is an efficient tool for the characterization of corrosion protection nanolayers on copper alloys and stainless steel. in 'Proceedings of the International Conference Nanomaterials: Applications and Properties. Alushta, Ukraine, Sumy State University', paper no. 01PCSI04, 1-3 (2013)

24 Erbil H. Y., Evaporation of pure liquid sessile and spherical suspended drops: A review. Advances in Colloid and Interface Science, 170(1-2), 67-86 (2012).

DOI: $10.1016 /$ j.cis.2011.12.006

25 Hoque E., DeRose J. A., Bhusan B, Hipps K. W., Low adhesion, non-wetting phosphonate self-assembled monolayer films formed on copper oxide surfaces. Ultramicroscopy, 109(8), 1015-1022 (2009). DOI: 10.1016/j.ultramic.2009.03.033

26 Surface Energy Data for PS: Polystyrene, CAS \#9003-53-6. Diversified Enterprises (2009).

http://www.accudynetest.com/polymer_surface_data/polystyrene.pdf (16 November 2013) 